\title{
Portable visible and near-infrared spectrophotometer for triglyceride measurements
}

\author{
TAKANORI KOBAYASHI ${ }^{1,2}$, YUKIKO HAKARIYA KATO ${ }^{1,2}$, \\ MEGUMI TSUKAMOTO $^{1}$, KAZUYOSHI IKUTA ${ }^{1}$ and AKIKAZU SAKUDO ${ }^{1,2}$ \\ ${ }^{1}$ Department of Virology, Center for Infectious Disease Control, Research Institute for Microbial Diseases, \\ Osaka University, Yamadaoka, Suita, Osaka 565-0871; ${ }^{2}$ Department of Physiology, \\ Osaka City University Graduate School of Medicine, Abeno-ku, Osaka 545-8585, Japan
}

Received August 25, 2008; Accepted October 20, 2008

DOI: $10.3892 /$ ijmm_00000103

\begin{abstract}
An affordable and portable machine is required for the practical use of visible and near-infrared (Vis-NIR) spectroscopy. A portable fruit tester comprising a Vis-NIR spectrophotometer was modified for use in the transmittance mode and employed to quantify triglyceride levels in serum in combination with a chemometric analysis. Transmittance spectra collected in the 600 - to 1100 -nm region were subjected to a partial least-squares regression analysis and leave-out cross-validation to develop a chemometrics model for predicting triglyceride concentrations in serum. The model yielded a coefficient of determination in cross-validation $\left(R_{\mathrm{VAL}}^{2}\right)$ of 0.7831 with a standard error of cross-validation (SECV) of $43.68 \mathrm{mg} / \mathrm{dl}$. The detection limit of the model was $148.79 \mathrm{mg} / \mathrm{dl}$. Furthermore, masked samples predicted by the model yielded a coefficient of determination in prediction $\left(R_{\text {PRED }}^{2}\right)$ of 0.6856 with a standard error of prediction (SEP) and detection limit of 61.54 and $159.38 \mathrm{mg} / \mathrm{dl}$, respectively. The portable Vis-NIR spectrophotometer may prove convenient for the measurement of triglyceride concentrations in serum, although before practical use there remain obstacles, which are discussed.
\end{abstract}

\section{Introduction}

Blood tests are important for the diagnosis and treatment of diseases. Currently, levels of blood constituents are mainly determined by biochemical analysis. The values obtained

Correspondence to: Dr Akikazu Sakudo, Department of Virology, Center for Infectious Disease Control, Research Institute for Microbial Diseases, Osaka University, 3-1 Yamadaoka, Suita, Osaka 565-0871, Japan

E-mail:sakudo@biken.osaka-u.ac.jp

Key words: visible and near-infrared, triglyceride, chemometrics, spectra provide a clue as to the health status of an individual. For example, an increased level of triglyceride in blood implies a high risk for atherosclerosis and is also linked to the risk of heart disease and stroke (1). Excessive levels of triglyceride $(>500 \mathrm{mg} / \mathrm{dl})$ are also reported to cause inflammation of the pancreas (pancreatitis) (2). The condition for which levels of glycerides including monoglycerides, diglycerides, and triglycerides are high is known as 'hyperglyceridemia' or 'hyperglyceridaemia' $(1,2)$. Triglycerides are present in most organs, but are mainly produced in fat, the liver and the intestines (3). Triglyceride is mainly stored in fat, and plasma triglyceride is a component of very low density lipoprotein (VLDL). Triglyceride plays an important role in metabolism as a source of energy and as a transporter of dietary fat (3).

The development of analytical methods for testing blood is even more important with the increasing relevance of health care for promoting quality of life. Although a biochemical analysis employing an automated system has been established for the detection of blood constituents including triglyceride (4), the method is yet to be perfected in terms of the consumption of reagents (5), which means that a specific substrate is required for determining each constituent. Furthermore, with increased knowledge regarding disease and health, the number of constituents which should be checked is increasing. Therefore, an improved clinical analysis which is cost-effective is required.

Visible and near-infrared (Vis-NIR) spectroscopy is a fast multicomponent assay, which makes possible the simultaneous analysis of various constituents. The other advantage of this method is that there is no requirement for sample preparations and reagents (6). This provides a high cost-effectiveness. On the other hand, as the Vis-NIR region has shown relatively weak water absorption with low energy levels (7), Vis-NIR spectra usually are not directly used for analysis and instead are applied to chemometric analysis for locating important wavelengths of certain constituents in blood.

The Vis-NIR region is dominated by weak overtones and combinations of vibration bands of atoms with strong molecular bonds containing $\mathrm{C}-\mathrm{H}, \mathrm{N}-\mathrm{H}$ and $\mathrm{O}-\mathrm{H}$ (8). As most biomolecules contain $\mathrm{C}-\mathrm{H}, \mathrm{N}-\mathrm{H}$ and $\mathrm{O}-\mathrm{H}$, this method is useful for the study of biological samples. It is therefore not surprising that Vis-NIR spectroscopy is employed extensively in the agricultural, pharmaceutical, chemical, petrochemical, 

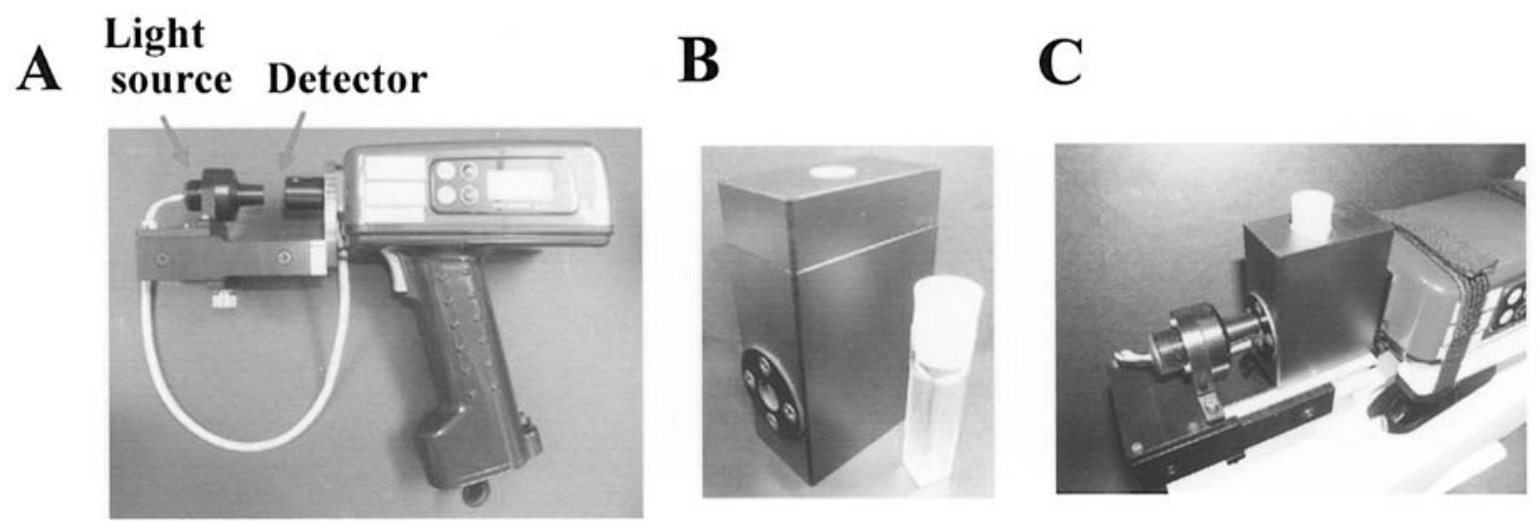

Figure 1. Schematic representation of the measurement of serum samples by transmittance mode using a modified portable spectrophotometer (FQANIRGUN). (A) Light source and detector in the modified FQA-NIRGUN, which was used for transmittance, (B) the polystyrene cuvette and cuvette folder, and $(\mathrm{C})$ the polystyrene cuvette and cuvette folder attached to the modified FQA-NIRGUN.

and medical fields (6-16). Vis-NIR spectroscopy has also been used to study constituents in blood such as glucose $(17,18)$, globulin $(17,19)$, protein $(19,20)$, urea $(17,19)$, B-lipoprotein $(20)$, triglycerides $(17,19)$ and albumin $(17,19)$. Although good results were obtained, highly sensitive and expensive machinery was needed. For this method to be practical, an affordable and portable Vis-NIR machine is required. Therefore, in this study, a portable Vis-NIR device for clinical analysis has been exploited for triglycerides in blood in combination with a partial least squares regression (PLSR) analysis by modifying a currently used fruit tester.

\section{Materials and methods}

Serum samples. Blood was collected from the antecubital vein. As triglyceride levels increase temporarily after eating, the blood samples were taken after fasting. Sera from 45 volunteers (41.20 \pm 11.10 years of age; male/female: $21 / 24)$ were used as test samples to develop a calibration model of PLSR, whereas another 132 determinations from other volunteers (39.55 \pm 9.47 years of age; male/female: $23 / 21)$ were masked and used for prediction. This research project was approved by the Ethics Committee of Osaka City University, and written informed consent was obtained from all volunteers. All samples were diluted 10-fold with phosphatebuffered saline (PBS) and adjusted to a constant volume (2 ml) in a polystyrene cuvette before Vis-NIR spectroscopy.

Vis-NIR instruments and measurements. An FQA (Fruit quality analyzer)-NIRGUN spectrophotometer (Japan Fantec Research Institute, Shizuoka, Japan), routinely used for testing fruits in the interactance mode, was modified for measuring the transmittance of serum samples. The detector was attached opposite the light source, enabling transmittance measurements (Fig. 1). Transmittance spectra of the serum samples in polystyrene cuvettes (optical length $10 \mathrm{~mm}$ ) (Sarstedt, Numbrecht, Germany) were measured at a 2-nm resolution with the modified FQA-NIRGUN spectrophotometer in an air-conditioned room at $37^{\circ} \mathrm{C}$. Spectral data were collected to yield absorbance values $[\log (1 / T)]$, where $T$ is the transmittance measured within a wavelength of 600-1100 nm. Three consecutive spectra were taken from each sample.
Data processing and chemometrics. Pirouette software (ver. 3.11; Infometrix, Woodinville, WA) was employed for data processing. To minimize differences between spectra caused by baseline shifts and noise prior to calibration, spectral data were mean-centered and transformed by smoothing based on the Savitsky-Golay algorithm (21). The mathematical formulas are available in the Pirouette manual. The PLSR method was used to develop a PLSR model for predicting triglyceride concentration. The number of PLSR factors was chosen from the minimized standard error of cross-validation (SECV). Calculations were performed by leave-out cross validation. The accuracy of the model was evaluated using SECV, while the standard deviation (SD) divided by the SECV and the coefficient of determination in cross-validation $\left(R^{2}{ }_{\mathrm{VAL}}\right)$ were determined accordingly. Furthermore, to evaluate the efficacy of the PLSR model using masked samples, which were not used to develop the model, the standard error of prediction (SEP), SD divided by SEP, and the coefficient of determination in prediction $\left(R_{\text {PRED }}^{2}\right)$ were calculated.

Calculation of the detection limit. The limit of detection was calculated according to Miller (22). Equation 1 defines the detection limit (y) that indicates if a sample contained a certain triglyceride based on the average $\left(\mathrm{y}_{\mathrm{B}}\right)$ and standard deviation $\left(S_{B}\right)$ of the signal from the blank control (signal of hypothetical tissues without triglyceride). The calculated intercept is used as an estimate of $y_{B}$. Equation 2 is used to estimate $S_{B}$, or statistics $S_{\mathrm{y} / \mathrm{x}}$, which estimates random errors in the y-direction (22). The $y_{i}^{\prime}$ values are the points corresponding to the individual $\mathrm{x}$-values on the calculated regression line:

$$
\begin{array}{cc}
y=y_{B}+3 S_{B} & \text { (Equation 1) } \\
S_{y / x}=\left\{\frac{\sum_{i}\left(y_{i}-y_{i}^{\prime}\right)^{2}}{n-2}\right\}^{1 / 2} & \text { (Equation 2) }
\end{array}
$$

Biochemical measurement of triglyceride. An aliquot of $0.5 \mathrm{ml}$ of each serum sample was subjected to biochemical measurements of triglycerides. The analysis was performed with the L-type Wako-TG-H Kit (Wako, Osaka, Japan) based on the manufacturer's instructions using the Clinical Analyzer 7700 (Hitachi High-Technologies Corp., Tokyo, Japan). 


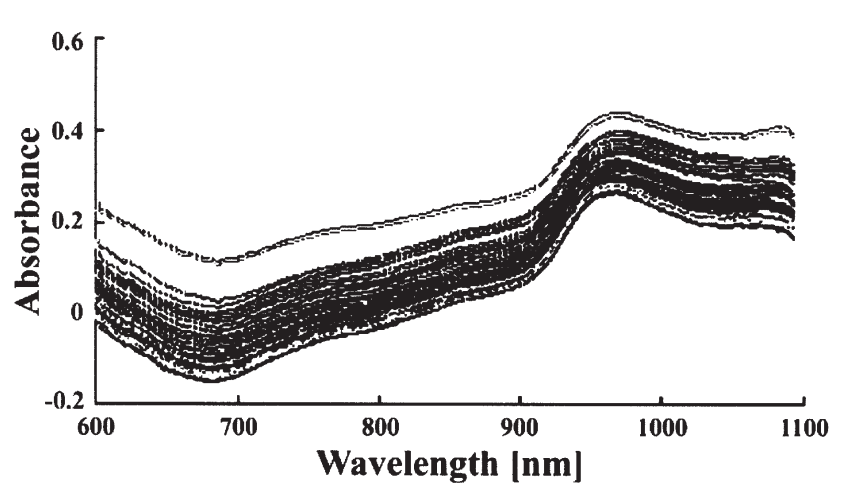

Figure 2. Three consecutive transmittance spectra of serum samples collected by the modified FQA-NIRGUN.

\section{Results and discussion}

The transmittance spectra of serum samples were collected at wavelengths of $600-1100 \mathrm{~nm}$. Fig. 2 shows 3 consecutive smoothed Vis-NIR spectra of all serum samples used in this study. The overall appearance of the $\log (1 / T)$ spectral curve has a concave character. These spectra were very similar to those for water and PBS (data not shown). Therefore, this shape is due to the various absorption bands of water. This is consistent with the report that the peak near $970 \mathrm{~nm}$ was previously assigned to the second overtone of the O-Hstretching mode $(6,8,23)$.

Next, Vis-NIR spectra were examined using chemometrics, while triglyceride levels were also determined biochemically with an automated clinical analyzer. The concentrations of serum samples covered ranges of triglycerides for normal and abnormal levels $(38-572 \mathrm{mg} / \mathrm{dl})$. The transmittance spectra were then used for constructing PLSR models for predicting triglyceride concentrations. To determine the predominant changes in the spectra, two forms of preprocessing were performed; viz., mean-centering and smoothening. Mean-centering normalized the baseline variations in the spectra, while smoothing minimized variations in noise. Following the determination of the predominant species in the spectra, a PLSR analysis of pre-processed data was performed. A full cross-validation was then applied to construct the PLSR calibration models. The SECV of triglyceride reached a minimum with a factor of 9 . This means that the optimal number of PLSR factors was 9. Good regression plots and regression coefficients of the PLSR calibration model for predicting triglyceride concentrations are shown in Fig. 3A and $\mathrm{C}$.

A good correlation in masked samples (triglyceride concentration range $42-448 \mathrm{mg} / \mathrm{dl}$ ) was also obtained between triglyceride values and predicted values based on Vis-NIR spectroscopy (Fig. 3B). The correlation between the data and line identity of triglyceride yielded an $R^{2}$ VAL value of 0.7830 and $R_{\text {PRED }}^{2}$ value of 0.6856 . The SECV in the test and masked samples was calculated to be 43.68 and $103.56 \mathrm{mg} /$ $\mathrm{dl}$, respectively. The calibration model meets the criteria, $R_{\mathrm{VAL}}^{2}>0.70$, in terms of the coefficient (24). Even $R_{\text {PRED }}^{2}$, the value of the correlation coefficient, was close to 0.70 . Furthermore, when the model was assessed by other criteria (poor when SD/SECV was <1.6; acceptable at 1.6-2.0; and excellent at $>2.0)(25)$, SD/SECV provided reliable results; viz., 1.9474 for test samples and 1.6830 for masked samples. Therefore, the present study used wavelengths of $600-1100 \mathrm{~nm}$ to generate an acceptable model for predicting triglyceride concentrations, even though a portable Vis-NIR spectrophotometer was used for collecting spectra. Based on Equation 1, the detection limits of the model were also calculated. The respective detection limits for test and masked samples were 148.79 and $159.38 \mathrm{mg} / \mathrm{dl}$. According to guidelines of the American Heart Association (26), the level of triglyceride $(\mathrm{mg} / \mathrm{dl})$ and the interpretation are as follows: $<150 \mathrm{mg} / \mathrm{dl}$ is normal and represents the lowest risk, $150-199 \mathrm{mg} / \mathrm{dl}$ is borderline high, and 200-499 mg/dl is high. More than $500 \mathrm{mg} /$ $\mathrm{dl}$ is very high and represents an increased risk. Therefore, this PLSR model would be applicable to judge if individuals have normal or high risk levels of triglyceride using serum samples.

The PLSR model provides not only for the development of quantitative models but also for the spectroscopic characterization of samples. Next, to examine the spectroscopic characterization of triglyceride in blood, the regression coefficient for the PLSR model was investigated (Fig. 3C). A line plot of this object reveals the independent variables
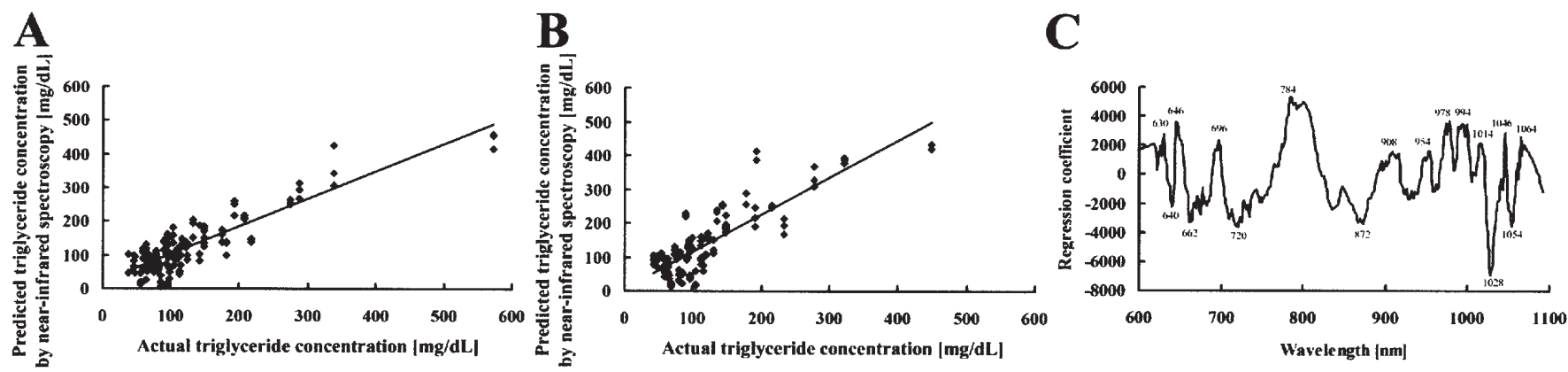

Figure 3. PLSR calibration models and prediction of triglyceride concentrations in sera using the modified FQA-NIRGUN. (A) Cross-validation model for triglyceride concentrations: 9 PLSR factors based on a 600 - to $1100-\mathrm{nm}$ region using a leave-out cross validation procedure. $R^{2}{ }_{\mathrm{VAL}}=0.7830$, SECV $=43.68 \mathrm{mg} /$ $\mathrm{dl}, \mathrm{SD}=86.22 \mathrm{mg} / \mathrm{dl}, \mathrm{SD} / \mathrm{SECV}=1.9474$ and detection limit=148.79 $\mathrm{mg} / \mathrm{dl}$. (B) Predictions of triglyceride concentrations of masked samples by the PLSR model. $R_{\text {PRED }}^{2}=0.6856, \mathrm{SEP}=61.54 \mathrm{mg} / \mathrm{dl}, \mathrm{SD}=103.56 \mathrm{mg} / \mathrm{dl}, \mathrm{SD} / \mathrm{SEP}=1.6830$ and detection limit=159.38 mg/dl. (C) Regression coefficient for the PLSR model of (A). Peak wavelengths are indicated accordingly and also summarized in Table I. 
Table I. The possible assignment of the peaks in the regression coefficient of the PLSR model shown in Fig. 3.

\begin{tabular}{|c|c|c|}
\hline Wavelength (nm) & Assignment & References \\
\hline$\uparrow 630$ & $\mathrm{~N}-\mathrm{H}$ stretch, 4th overtone of amides (primary, bonded; two bonds) $(630 \mathrm{~nm})$ & $(23)$ \\
\hline$\downarrow 640$ & $\mathrm{~N}-\mathrm{H}$ stretch, 4th overtone of amides (secondary, bonded; one band) (600-640 nm) & $(23)$ \\
\hline$\uparrow 646$ & Unknown & - \\
\hline$\downarrow 662$ & Unknown & - \\
\hline$\uparrow 696$ & Unknown & - \\
\hline$\downarrow 720$ & $\mathrm{OH}$ stretch, 3rd overtone of $\mathrm{ROH}(738 \mathrm{~nm})$ & $(6,8)$ \\
\hline \multirow[t]{3}{*}{$\uparrow 784$} & N-H stretch, 3rd overtone of amides (primary, bonded; two bonds) $(\sim 788 \mathrm{~nm})$ & $(23)$ \\
\hline & $\mathrm{N}-\mathrm{H}$ stretch, 3rd overtone of $\mathrm{RNH}_{2}(779 \mathrm{~nm})$ & $(6,8)$ \\
\hline & $\mathrm{N}-\mathrm{H}$ stretch, 3rd overtone of amides (secondary, bonded; one band) $(750-800 \mathrm{~nm}$ ) & $(23)$ \\
\hline$\downarrow 872$ & C-H stretch, 3rd overtone of ArCH. (aromatics) $(857-890 \mathrm{~nm})$ & $(23)$ \\
\hline$\uparrow 908$ & C-H stretch, 3rd overtone of protein $(910 \mathrm{~nm})$ & $(6,8)$ \\
\hline$\uparrow 954$ & O-H stretch, 2nd overtone of ROH (alcohols) (940-970 nm) & (23) \\
\hline \multirow[t]{4}{*}{$\uparrow 978$} & $\mathrm{O}-\mathrm{H}$ stretch, 2nd overtone of $\mathrm{ROH}$ and $\mathrm{H}_{2} \mathrm{O}(970 \mathrm{~nm})$ & $(6,8)$ \\
\hline & $\mathrm{O}-\mathrm{H}$ stretch, 2nd overtone of $\mathrm{H}_{2} \mathrm{O}(960-970 \mathrm{~nm})$ & $(23)$ \\
\hline & N-H stretch, 2nd overtone of .CONHR (secondary amides) (981 nm) & $(23)$ \\
\hline & $\mathrm{N}-\mathrm{H}$ stretch, 2nd overtone of . $\mathrm{CONH}_{2}$ (primary amide) $(975-989 \mathrm{~nm})$ & $(23)$ \\
\hline \multirow[t]{2}{*}{$\uparrow 994$} & $\mathrm{~N}-\mathrm{H}$ stretch, 2nd overtone of $\mathrm{ArNH}_{2}$ (aromatic amines) $(995 \mathrm{~nm})$ & $(23)$ \\
\hline & N-H stretch, 2nd overtone of .NH (amines, general) (1000 nm) & $(23)$ \\
\hline \multirow[t]{3}{*}{$\uparrow 1014$} & $2 X \mathrm{~N}-\mathrm{H}$ stretch $+2 \mathrm{X}$ amide $\mathrm{I}$ (protein) $(1020 \mathrm{~nm})$ & $(23)$ \\
\hline & N-H stretch, 2nd overtone (protein) (1007 nm) & $(23)$ \\
\hline & $\mathrm{N}-\mathrm{H}$ stretch, combination of $\mathrm{RNH}_{2}$ (primary amine) $(1020 \mathrm{~nm})$ & $(23)$ \\
\hline$\downarrow 1028$ & $\mathrm{~N}-\mathrm{H}$ stretch, 2nd overtone of $\mathrm{RNH}_{2}(1030 \mathrm{~nm})$ & $(23)$ \\
\hline \multirow[t]{3}{*}{$\uparrow 1046$} & N-H stretch, 2nd overtone of amides (primary, bonded; two bonds) $(\sim 1050 \mathrm{~nm})$ & $(23)$ \\
\hline & $\mathrm{N}-\mathrm{H}$ stretch, combination of CONH (primary amide) $(1047 \mathrm{~nm})$ & $(23)$ \\
\hline & $\mathrm{N}-\mathrm{H}$ stretch, 3rd overtone of amides (secondary, bonded; one band) (1000-1067 nm) & $(23)$ \\
\hline$\downarrow 1054$ & N-H stretch, 2nd overtone of $\mathrm{R}-\mathrm{NH}_{2}(1060 \mathrm{~nm})$ & $(6,8)$ \\
\hline$\uparrow 1064$ & $\mathrm{~N}-\mathrm{H}$ stretch, 2nd overtone of $\mathrm{R}-\mathrm{NH}_{2}(1060 \mathrm{~nm})$ & $(6,8)$ \\
\hline
\end{tabular}

$\uparrow$ positive peak; $\downarrow$ negative peak.

(wavelength) important in modeling the dependent variable (triglyceride concentration). A list of peaks in the regression coefficients in this model is shown in Table I. Many peaks near 630, 640, 646, 662, 696, 720, 784, 872, 908, 954, 978, $994,1014,1028,1046,1054$ and $1064 \mathrm{~nm}$ were observed in the loading of regression coefficients of triglyceride in blood. Although it is often difficult to assign wavelengths to specific molecular absorptions in the Vis-NIR region, the possible assignment of each peak is also shown in Table I. Most of the observed peaks seem to be related to interactions between triglyceride and water or other organic compounds including proteins in blood. This is because several peaks related to proteins, amide, and amine were observed at 630, 640, 784, 908, 978, 994, 1014, 1028, 1046, 1054 and $1064 \mathrm{~nm}(6,8,23)$, while those near $978 \mathrm{~nm}$ were indicative of water (Table I) (23). Moreover, peaks related to aromatics and alcohols were also observed at 720, 872, 954 and $978 \mathrm{~nm}$ (Table I) $(6,8,23)$. This is consistent with the notion that the concentration of triglyceride is measured indirectly by absorbance corresponding to the specific interaction between water or organic compounds.

The current methods for clinical analysis in blood are biochemical. It is worth noting that this approach is destructive. Recently, the use of Vis-NIR spectroscopy to quantify blood constituents has been studied. However, the instrumentation needed is expensive. The results of our study suggest that a portable Vis-NIR device provides a non-destructive means of monitoring triglyceride levels in blood. Other advantages include an affordable price (1.5 million yen), prompt analysis, simplicity in sample preparations, and no requirements for chemical reagents. Therefore, Vis-NIR spectroscopy could be a convenient assay for measuring triglyceride concentrations in blood. However, as previous studies have reported that the control of key physical and chemical parameters such as temperature is required for accuracy and precision (18), possible limitations should be further studied before the practical use of this method. Studies using this and other 
Vis-NIR instruments for detecting other blood constituents and diagnosing diseases $(10,11,27)$ are ongoing.

\section{Acknowledgements}

We thank Dr Hirohiko Kuratsune (Fatigue Clinical Center, 21st Century COE Program, Osaka City University Graduate School of Medicine, Osaka, Japan) for discussions and the kind help of collecting blood from volunteers. The authors are grateful to Yukiyoshi Hirase (Sakai Bio-Clinical Laboratory, Osaka, Japan) for the invaluable comments and help. This study was partly supported by Grants-in-Aid from Heiwa Nakajima Foundation, and Japan Science and Technology Agency and Kieikai Research Foundation (Tokyo, Japan).

\section{References}

1. Malloy MJ and Kane JP: A risk factor for atherosclerosis: triglyceride-rich lipoproteins. Adv Intern Med 47: 111-136, 2001.

2. Oh RC and Lanier JB: Management of hypertriglyceridemia. Am Fam Physician 75: 1365-1371, 2007.

3. Nikkila EA, Taskinen MR and Sane T: Plasma high-density lipoprotein concentration and subfraction distribution in relation to triglyceride metabolism. Am Heart J 113: 543-548, 1987.

4. Warnick GR: Measurement of cholesterol and other lipoprotein constituents in the clinical laboratory. Clin Chem Lab Med 38 287-300, 2000

5. Janatsch G, Kruse-Jarres JD, Marbach R and Heise HM: Multivariate calibration for assays in clinical chemistry using attenuated total reflection infrared spectra of human blood plasma. Anal Chem 61: 2016-2023, 1989.

6. Osborne BG and Fearn T: Near-Infrared Spectroscopy in Food Analysis. Longman Scientific \& Technical, UK, 1986.

7. Ciurczak EW and Drennen JK: Pharmaceutical and Medical Applications of Near-Infrared Applications (Practical Spectroscopy). Marcel Dekker Inc., New York, 2002.

8. Osborne BG, Fearn T and Hindle PT: Practical NIR Spectroscopy With Applications in Food and Beverage Analysis (Longman Food Technology). Longman Group United Kingdom, UK 1993.

9. Raghavachari R: Near-Infrared Applications in Biotechnology (Practical Spectroscopy). Marcel Dekker Inc., New York, 2001.

10. Sakudo A, Kuratsune H, Kobayashi T, Tajima S, Watanabe Y and Ikuta K: Spectroscopic diagnosis of chronic fatigue syndrome by visible and near-infrared spectroscopy in serum samples. Biochem Biophys Res Commun 345: 1513-1516, 2006.
11. Sakudo A, Suganuma Y, Kobayashi T, Onodera T and Ikuta K: Near-infrared spectroscopy: promising diagnostic tool for viral infections. Biochem Biophys Res Commun 341: 279-284, 2006.

12. Sakudo A, Tsenkova R, Onozuka T, et al: A novel diagnostic method for human immunodeficiency virus type-1 in plasma by near-infrared spectroscopy. Microbiol Immunol 49: 695-701, 2005.

13. Sakudo A, Tsenkova R, Tei K, Morita H, Ikuta K and Onodera T: Ex vivo tissue discrimination by visible and near-infrared spectra with chemometrics. J Vet Med Sci 68: 1375-1378, 2006.

14. Sakudo A, Tsenkova R, Tei K, et al: Comparison of the vibration mode of metals in $\mathrm{HNO}_{3}$ by a partial least-squares regression analysis of near-infrared spectra. Biosci Biotechnol Biochem 70: 1578-1583, 2006.

15. Sakudo A, Yoshimura E, Tsenkova R, Ikuta K and Onodera T: Native state of metals in non-digested tissues by partial least squares regression analysis of visible and near-infrared spectra. J Toxicol Sci 32: 135-141, 2007.

16. Nojima J, Sakudo A, Hakariya Y, et al: Spectroscopic diagnosis of anti-phospholipid antibodies by visible and near-infrared spectroscopy in SLE patients' plasma samples. Biochem Biophys Res Commun 362: 522-524, 2007.

17. Hall JW and Pollard A: Near-infrared spectrophotometry: a new dimension in clinical chemistry. Clin Chem 38: 1623-1631, 1992.

18. Pan S, Chung H, Arnold MA and Small GW: Near-infrared spectroscopic measurement of physiological glucose levels in variable matrices of protein and triglycerides. Anal Chem 68: 1124-1135, 1996.

19. Hall JW and Pollard A: Near-infrared spectroscopic determination of serum total proteins, albumin, globulins, and urea Clin Biochem 26: 483-490, 1993.

20. Domján G, Kaffka KJ, Jákó JM and Vályi-Nagy IT: Rapid analysis of whole blood and blood serum using near infrared spectroscopy. J Near Infrared Spectrosc 2: 67-78, 1994.

21. Savitzky A and Golay MJE: Smoothing and differentiation of data by simplified least squares procedures. Anal Chem 36: 1627-1639, 1964.

22. Miller JN and Miller JC: Statistics and chemometrics for analytical chemistry. Prentice Hall College Division, UK, 2000.

23. Workman J: Handbook of Organic Compounds: NIR, IR, Raman and UV-Vis Spectra Featuring Polymers and Surfactants. Academic Press, San Diego, 2001.

24. Sauvage L, Frank D, Stearne J and Millikan MB: Trace metal studies of selected white wines: An alternative approach. Anal Chim Acta 458: 223-230, 2002.

25. Moron A and Cozzolino D: Exploring the use of near-infrared reflectance spectroscopy to study physical properties and microelements in solid. J Near Infrared Spectrosc 11: 145-154, 2003.

26. American Heart Association: Triglycerides. http://www/ americanheart.org/presenter.jhtml?identifier $=4778$.

27. Kato YH, Matsunaga H, Sakudo A and Ikuta K: Visible and near-infrared spectral changes in plasma of psychiatric patients. Int J Mol Med 22: 513-519, 2008. 